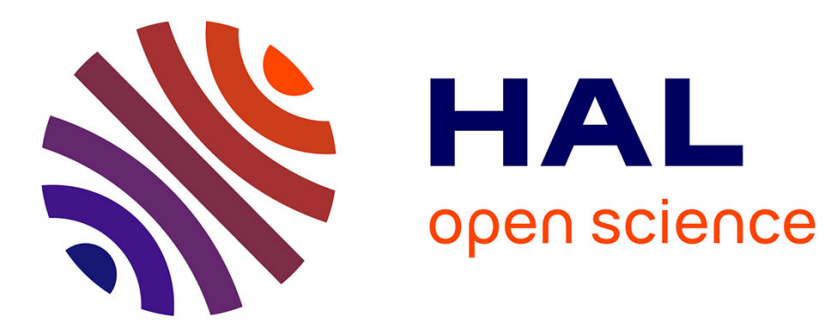

\title{
Exciting Wide NMR Spectra of Static Solid Samples with Weak Radiofrequency Fields
}

Diego Carnevale, Srinivas Chinthalapalli, Geoffrey Bodenhausen

\section{To cite this version:}

Diego Carnevale, Srinivas Chinthalapalli, Geoffrey Bodenhausen. Exciting Wide NMR Spectra of Static Solid Samples with Weak Radiofrequency Fields. Zeitschrift für Physikalische Chemie, 2017, 231 (3), pp.527-543. 10.1515/zpch-2016-0808 . hal-01501410

\section{HAL Id: hal-01501410 https://hal.sorbonne-universite.fr/hal-01501410}

Submitted on 4 Apr 2017

HAL is a multi-disciplinary open access archive for the deposit and dissemination of scientific research documents, whether they are published or not. The documents may come from teaching and research institutions in France or abroad, or from public or private research centers.
L'archive ouverte pluridisciplinaire HAL, est destinée au dépôt et à la diffusion de documents scientifiques de niveau recherche, publiés ou non, émanant des établissements d'enseignement et de recherche français ou étrangers, des laboratoires publics ou privés. 


\section{Exciting wide NMR spectra of static solid samples with weak radiofrequency fields}

Diego Carnevale, ${ }^{1,2, *}$ Srinivas Chinthalapalli ${ }^{2,3}$ and Geoffrey Bodenhausen ${ }^{2,4,5}$

${ }^{1}$ Neuchâtel Platform of Analytical Chemistry (NPAC), Institut de Chimie, Université de Neuchâtel, Avenue de Bellevaux 51, 2000 Neuchâtel, Switzerland

${ }^{2}$ Institut des sciences et ingénierie chimiques (ISIC), Ecole Polytechnique Fédérale de Lausanne (EPFL), 1015 Lausanne, Switzerland

${ }^{3}$ Analytical and Spectroscopy Division (ASD), Analytical, Spectroscopy and Ceramics Group (ASCG), Propellants, Polymers, Chemicals and Materials (PCM) Entity, Vikram Sarabhai Space Centre (VSSC), Thiruvananthapuram 695 022, India

${ }^{4}$ Departement de Chimie, Ecole Normale Superieure, PSL Research University, UPMC Univ Paris 06, CNRS, Laboratoire des Biomolecules (LBM), 24 rue Lhomond, 75005 Paris, France

${ }^{5}$ Sorbonne Universites, UPMC Univ Paris 06, Ecole Normale Superieure, CNRS, Laboratoire des Biomolecules (LBM), Paris, France

Corresponding author: *Diego Carnevale diego.carnevale@unine.ch

To be submitted to: Zeitschrift für Physikalische Chemie 


\begin{abstract}
Trains of short pulses in the manner of 'delays alternating with nutations for tailored excitation' (DANTE) have been applied to the Pake patterns of protons of water molecules trapped in a static powdered sample of barium chlorate monohydrate. The spin dynamics in the course of such experiments have been investigated by means of numerical simulations and compared with the ideal refocusing that can be achieved under magic-angle spinning. Solid echoes yield essentially undistorted lineshapes, in contrast to direct excitation without refocusing that leads to severe dispersions of the phases because of inhomogeneous interactions such as homonuclear dipolar couplings and anisotropic chemical shifts. The selectivity of DANTE sequences allows one to access 'slices' of the Pake pattern that can be related to particular crystallite orientations. Single-crystal spectra can therefore be extracted from powder spectra. A similar behavior is expected for both dipolar and quadrupolar echoes.
\end{abstract}

\title{
Key words
}

NMR Spectroscopy, Solid-State NMR of Static Samples, DANTE, Hydration Water, Dipolar Interactions, Quadrupolar Interactions, Dipolar Echoes, Quadrupolar Echoes, Solid Echoes.

\author{
Abbreviations \\ Delays Alternating with Nutations for Tailored Excitation (DANTE) \\ Single-Quantum (SQ) \\ Coherence Transfer Pathway (CTP) \\ Magic Angle Spinning (MAS)
}




\section{Introduction}

Solid-state NMR is an invaluable tool for the structural characterization of systems where diffraction techniques are less effective due to dynamics or to a lack of long-range order. Valuable insight into dynamic processes such as chemical exchange and molecular reorientation can be obtained. When the interactions that affect nuclear spins in solids are anisotropic,[1] this results in static NMR lineshapes commonly referred to as 'powder patterns' that are inhomogeneously broadened. In samples spinning about the magic-angle, the properties of DANTE trains [2,3] of radiofrequency $(r f)$ pulses applied in a rotor-synchronized fashion have been thoroughly investigated.[4-8] In this paper, we turn our attention to the application of such pulse trains to static samples that are subject to inhomogeneous broadening. DANTE sequences can offer both a uniform broadband excitation extending over several $\mathrm{MHz}$, and the selective excitation of chosen frequency ranges extending over a few $\mathrm{kHz}$. These paradoxical features are particularly appealing to study samples that are subject to large inhomogeneous interactions, such as hyperfine couplings in paramagnetic systems, or dipolar interactions.[6,7] For the latter interactions, a spin system comprising only isolated pairs of nuclear spins, like water trapped in gypsum $\left(\mathrm{CaSO}_{4}\right)[10]$ offers an instructive example. We have investigated the protons of water trapped in barium chlorate monohydrate, $\mathrm{Ba}\left(\mathrm{ClO}_{3}\right)_{2} \cdot \mathrm{H}_{2} \mathrm{O}$.[9] The distance between the two protons belonging to a given water molecule is ca. $1.6 \AA$, leading to a homonuclear dipolar coupling constant of ca. $30 \mathrm{kHz}$, giving rise to a nearly ideal Pake pattern [10]. The distance between two neighboring water molecules in $\mathrm{Ba}\left(\mathrm{ClO}_{3}\right)_{2} \cdot \mathrm{H}_{2} \mathrm{O}$ is ca. $4.8 \AA$, resulting in intermolecular dipolar couplings below $1 \mathrm{kHz}$ which barely affect the Pake pattern.

If one combines DANTE pulse trains with sample rotation about the magic angle, there is a synergy between the mechanical rotation, which refocuses the spatial parts of anisotropic interactions of rank 2 (but not of second-order quadrupolar couplings) to form rotational echoes, and the nutation due to the $r f$ pulses that affects the spin part of the interactions.[6] Neglecting homogeneous losses, this allows one to excite a coherence (transverse magnetization) through the cumulative effects of small flip angles in a uniform manner over several $\mathrm{MHz}$, provided all inhomogeneous interactions can be refocused by rotation about the magic angle. 
In static samples, on the other hand, there are of course no rotational echoes, and it is far from obvious that the cumulative effects of a train of pulses can excite a coherence. In this study, we investigate some properties of both excitation and refocusing by DANTE pulse trains applied to static samples. The spin dynamics that occur during the pulse trains are analyzed by means of numerical simulations. The refocusing properties of DANTE echoes in static samples are compared with echoes of equivalent experiments performed under magic-angle spinning (MAS), where full refocusing can be achieved. The comparison serves as a test for our experiments in static samples. Applications to the Pake pattern of $\mathrm{Ba}\left(\mathrm{ClO}_{3}\right)_{2} \cdot \mathrm{H}_{2} \mathrm{O}$ show how DANTE trains can be used to extract spectra corresponding to single crystals with different orientations with respect to the external magnetic field.

\section{Results and discussion}

In previous studies we have introduced the nomenclature $D_{N}^{K}$ to describe DANTE pulse trains applied to samples that are spinning at the magic angle, $N$ being the number of $r f$ pulses per rotor period and $K$ the number of rotor periods that corresponds to the length of the entire pulse train, which therefore comprises $M=K \times N$ pulses.[6] In static samples, where the concept of a rotor period is meaningless, a DANTE train $\left[\tau_{p}^{\phi}-\tau-\right]_{M}$ can simply be described by $D_{M}, M$ being the number of pulses that constitute the train. In this work, we will use the nomenclature $D_{M}^{\phi}$ where the index $\phi$ indicates the phase that is common to all $r f$ pulses in the pulse train. By analogy, we will use $D_{N}^{K, \phi}$ for spinning samples. Figure 1(a) depicts a pulse scheme inspired by the wellknown $90_{x}^{\circ}-\tau-90_{\phi}^{\circ}-\tau-$ scheme used to excite solid echoes with $\phi=\pi / 2 .[11,12]$ By themselves, such experiments cannot simultaneously refocus both linear interactions (chemical shifts) and quadratic interactions (dipolar or quadrupolar couplings).[13] This can however be achieved by combining a solid echo sequence with a suitable prescription for cycling the phase $\phi$ [14]. By analogy to the familiar $90_{x}^{\circ}-\tau-90_{\phi}^{\circ}-\tau$ - solid echo scheme, one can use a $D_{10}^{x}-\tau^{\prime}-D_{10}^{\phi}-\tau^{\prime}-$ sequence (or $\left[\tau_{p}^{x}-\tau-\right]_{M}-\tau^{\prime}-\left[\tau_{p}^{\phi}-\tau-\right]_{M}-\tau^{\prime}-$ ) comprising two DANTE combs, both with $M=10$, a delay between the two DANTE trains that may be set to $\tau^{\prime}=0$, and step the relative phase $\phi$ of the two $D_{10}^{\phi}$ blocks to refocus both chemical shifts and dipolar or 
quadrupolar interactions. To this effect, one must select the coherence transfer pathway $p=0 \rightarrow$ $p=+1 \rightarrow p=-1$. [13] The length $\tau_{p}$ of the individual pulses must be $\tau_{p}=\tau_{90^{\circ}} / M$, or, equivalently, their nutation angles must be $\beta=2 \pi v_{1} \tau_{90^{\circ}} / M=\pi /(2 M)$. If the individual pulses have a cumulative effect, each $D_{10}$ train corresponds to a $\pi / 2$ pulse, and the $D_{10}^{x}-\tau^{\prime}-D_{10}^{\phi}-\tau^{\prime}-$ sequence is therefore equivalent to a $90_{x}^{\circ}-\tau-90_{\phi}^{\circ}-\tau-$ solid echo sequence.

In order to understand the differences between static and spinning samples, we performed numerical simulations for a spin system comprising two equivalent dipolar-coupled $I=1 / 2$ spins. Figure 1 (b) shows a simulation of the behavior of single-quantum (SQ) coherences $\left\langle I_{ \pm}+S_{ \pm}\right\rangle$in a spinning sample in the course of the solid echo sequence $D_{1}^{10, x}-\tau^{\prime}-D_{1}^{10, \phi}-\tau^{\prime}-$ of Fig. 1(a), with an $r$-field strength $v_{1}=125 \mathrm{kHz}, \tau_{p}=0.2 \mu \mathrm{s}, M=10$, so that $M \tau_{p}=10 \times \tau_{p}=\tau_{90^{\circ}}=2 \mu \mathrm{s}$ for each $D_{10}$ block, $\tau_{\text {rot }}=33.3 \mu$ s corresponding to $v_{\text {rot }}=1 / \tau_{\text {rot }}=30.03 \mathrm{kHz}$, a delay between the two DANTE trains $\tau^{\prime}=0$, and intervals between adjacent pulses within each train of $\tau=\tau_{\text {rot }}-\tau_{p}=33.1 \mu \mathrm{s}$. Each $D_{10}$ block lasts $10 \times\left(\tau+\tau_{p}\right)=10 \times \tau_{\text {rot }}=333 \mu$ s. A chemical shift anisotropy (CSA) characterized by $\delta_{\text {iso }}=0 \mathrm{ppm}, \quad \Delta_{\text {aniso }}=-10 \quad \mathrm{ppm}$ and $\eta=$ 0.9 was included. The external field was $B_{0}=9.4 \mathrm{~T}(400 \mathrm{MHz}$ for protons) and 2000 crystal orientations were considered. For simplicity, the main axes of the shielding and dipolar tensors were assumed to be parallel to one another. All interactions are active during both intervals and rf pulses. In contrast to previous studies, we followed the coherences throughout the entire echo sequence. In the nutation profiles, a boost in signal intensity amounts to an increase of the observable single-quantum coherence or transverse magnetization. Coherence transfer selection may be imposed by Exorcycle [15], i.e., by incrementing the phase $\phi=n \Delta \phi$ (with $n=0,1,2,3$ and $\Delta \phi=\pi / 2$ ) of the second $D_{1}^{10, \phi}$ refocusing train, while alternately adding and subtracting the signals. As has been shown elsewhere for the excitation,[6] each pulse acts on a rotational echo so that a cumulative effect is observed, generating the full signal intensity corresponding to a $90^{\circ}$ pulse, i.e., at the end of the first $D_{1}^{10, x}$ DANTE train $\left\langle F_{-}\right\rangle=\operatorname{Tr}\left[\rho(t) F_{-}\right]=2$, where $\left\langle F_{ \pm}\right\rangle=\left\langle I_{ \pm}+S_{ \pm}\right\rangle$ . The second $D_{1}^{10, \phi}$ block leads to a build-up of coherence with order $p=-1$ by conversion from the coherence with order $p=+1$ generated by the first $D_{1}^{10, x}$ block, owing to the cumulative effect. 
Half-way during the sequence, provided coherence selection by phase cycling ensures that $p=+1$ $\rightarrow p=-1$, the signal drops to zero. This is due to the fact that $p=+1$ coherence (i.e., $\left.\left\langle F_{-}\right\rangle\right)$is selected during the first half of the echo whereas $p=-1$ (i.e., $\left.\left\langle F_{+}\right\rangle\right)$is selected during the second half of the echo. In other words, no $p=-1$ coherence is present after the first block $D_{1}^{10, x}$, since phase cycling cancels this coherence order. In contrast, during the second block $D_{1}^{10, \phi}, p=-1$ coherence is generated from the $p=+1$ coherence selected during the first block. At the end of the second $D_{1}^{10, \phi}$ block, the signal intensity is $\operatorname{Tr}\left[\rho(t) F_{+}\right]=1$, i.e., half of what was obtained after the first $D_{1}^{10, x}$ block. This stems from the fact that we consider a solid echo rather than a CarrPurcell echo. The dashed line in Fig. 1(b) passes through points at which the acquisition of FID's could by started (just before each pulse in the train) and indicates how the signal intensity of the resulting spectra would vary during such hypothetical experiments.

Figure 1(c) shows a profile analogous to that of Fig. 1(b) that one obtains for a static nonspinning sample with the sequence $D_{10}^{x}-\tau^{\prime}-D_{10}^{\phi}-\tau^{\prime}-$ with a delay between the two DANTE trains $\tau^{\prime}=0$. During the first $D_{10}^{x}$ train, as expected, no cumulative effect is observed since there are no rotational echoes. In the intervals between the pulses, the inhomogeneous interaction causes a dephasing of the $p=+1$ coherence and each pulse acts on partially dephased coherences, which, in contrast to spinning samples, results in a failure to build up any signal. As one can appreciate from Fig $1(\mathrm{~b})$ and $1(\mathrm{c})$, the signal intensity created by the first pulse is $\operatorname{Tr}\left[\rho(t) F_{-}\right]=$ 0.31 in both spinning and static samples.

Surprisingly, after the $p=+1 \rightarrow p=-1$ transfer, half-way during the sequence, one observes a completely different profile, with a cumulative effect that leads to a build-up of the signal similar to that of the spinning case. In the intervals between the pulses, one observes an increase in signal intensity. This is solely due to free evolution under inhomogeneous interactions and merely resembles the refocusing effect due to the mechanical rotation observed in the spinning case of Fig. 1(b). The dashed line has the same meaning as in Fig 1(b). It is interesting to note that, in contrast to the spinning case, where each pulse leads to a signal increase, in the static case each pulse results in a loss of signal during the second refocusing train. This decrease in intensity is 
however compensated by the above-mentioned increase due to the free evolution under the shift and dipolar interactions, resulting in a cumulative effect. In contrast to the spinning case, where the cumulative build-up is due to the action of the $r f$ pulses, in the static case the cumulative build-up during the second half of the echo is due to the refocusing of inhomogeneous broadenings ensured by coherence pathway selection. At the end of the $D_{10}^{x}-\tau^{\prime}-D_{10}^{\phi}-\tau^{\prime}-$ sequence, the signal intensity is $\operatorname{Tr}\left[\rho(t) F_{+}\right]=0.03$ in a static sample. Compared to the ideal fully refocused echoes that can be obtained in samples spinning about the magic angle, where the expectation value of the coherence at the top of the echo is $100 \%$, the fraction that can be refocused in static samples is only $3 \%$ in the spin system under investigation with our estimate of the average over all crystallite orientations in the powder. The profile of Fig. 1(b) corresponding to the second refocusing train of pulses has been amplified by a factor 4 .

Figures $1(d),(e)$ and (f) show, from left to right, the static spectra which would result if one started the signal acquisition directly after excitation (without refocusing), e.g., after pulse trains (d) $D_{1}^{x}-\tau^{\prime}-$, (e) $D_{5}^{x}-\tau^{\prime}-$ or (f) $D_{10}^{x}-\tau^{\prime}-$ (with $\tau^{\prime}=0$ ), as indicated by black arrows in Fig $1($ a). The spectra are highly distorted as a result of the dephasing that occurs during the $\tau$ intervals between pulses. A simulation of an ideal Pake pattern is overlaid in (f) with a vertical scaling factor. Figures $1(\mathrm{~g}),(\mathrm{h})$ and (i) show, from left to right, the spectra that would result if one acquired the signal after 'truncated' spin echo sequences (g) $D_{10}^{x}-\tau^{\prime}-D_{1}^{\phi}-\tau^{\prime}-$, $D_{10}^{x}-\tau^{\prime}-D_{5}^{\phi}-\tau^{\prime}-$ and (i) $D_{10}^{x}-\tau^{\prime}-D_{10}^{\phi}-\tau^{\prime}-$, respectively, as indicated by black arrows in Fig. 1(a) (with $\tau^{\prime}=0$ ). From left to right, one can appreciate the increase in signal intensity as the second DANTE train approaches a cumulative nutation angle of $90^{\circ}$. The spectrum of Fig. 1(i) results from a complete $D_{10}^{x}-\tau^{\prime}-D_{10}^{\phi}-\tau^{\prime}-$ solid echo sequence. As expected, one observes three peaks corresponding to three $r f$ spikelets spaced by $1 / \tau=30.03 \mathrm{kHz}$. Their envelope can be compared with the ideal static Pake pattern (red line) which extends over ca. $100 \mathrm{kHz}$. The intensities of the three peaks nicely reproduce that of the envelope of the static powder pattern.

In order to obtain insight into the spin dynamics during a DANTE echo sequence applied to a static sample, we shall consider the shortest possible DANTE sequence $D_{2}^{x}-\tau^{\prime}-D_{2}^{\phi}-\tau^{\prime}-$ that 
can be seen as analogous to a solid echo. For the sake of comparison with the ideal case of a complete refocusing, the MAS condition will also be considered. A scheme depicting the pulse sequence, with its corresponding coherence transfer pathway, is shown in Fig. 2(a), for $\tau^{\prime}=0$. The relevant state of the system is indicated by the index $(n)$. We chose a model system comprising two equivalent ${ }^{1} \mathrm{H}$ spins with a homonuclear dipolar coupling $d=-10 \mathrm{kHz}$, appropriate for a single crystal with a dipolar tensor perpendicular to the external magnetic field, and ideal pulses with flip angles $\beta=\pi / 4$ and delays between the pulses $\tau=100 \mu \mathrm{s}$. No shift interaction was included for simplicity. The profiles obtained, assuming proper selection of the change in coherence order, are shown in Fig 2(b) with black and red lines for spinning ( $\left.v_{\text {rot }}=10 \mathrm{kHz}\right)$ and static samples, respectively. Even with these drastic simplifications, the peculiar features highlighted in the profile of Fig 1(c) are preserved, i.e., the dephasing during the first train with $p$ $=0 \rightarrow p=+1$, the rephasing effect during the second train with $p=+1 \rightarrow p=-1$ and the ability of the dipolar interaction to increase the signal intensity under free evolution. This latter feature may be seen as a partial refocusing of the internal interactions. We monitored the relevant singlequantum matrix elements before and after each $r f$ pulse and at the end of the echo, as indicated by numbers in brackets in Fig 2(a). The state of the system at point $(n)$ of the pulse sequence is described by $\rho(n)=U_{\lambda} \rho(n-1) U_{\lambda}^{\dagger}$, with the propagator $U_{\lambda}=\exp \left(-i H_{\lambda} \tau\right)$ for the interaction $\lambda$, which can be either an rf-pulse or a dipolar coupling, described either by the Hamiltonian $H_{r f}=\omega_{1}\left(F_{x} \cos \phi+F_{y} \sin \phi\right)$ or by $H_{D}=-(d / 2)\left(3 \cos ^{2} \theta-1\right)\left(3 I_{z} S_{z}-I S\right)$. Since we make use of ideal (infinitely short) pulses, no interaction other than the $r f$ pulse can take place between states (2) and (3). Therefore, rather than 'at time $(n)^{\prime}$ ', we shall speak of 'at point $(n)$ ' of the pulse sequence shown in Fig. 2(a). In the spinning case, at points (2), (4), (6) and (8), a rotational echo is formed and the dipolar interaction is fully refocused by the mechanical rotation and can therefore be completely ignored. Therefore, in the spinning case it can be shown that $\left\langle F_{-}\right\rangle_{(1)}=\left\langle F_{-}\right\rangle_{(2)}=\sqrt{2}$, $\left\langle F_{-}\right\rangle_{(3)}=\left\langle F_{-}\right\rangle_{(4)}=2,\left\langle F_{+}\right\rangle_{(5)}=\left\langle F_{+}\right\rangle_{(6)}=1-1 / \sqrt{2}$ and $\left\langle F_{+}\right\rangle_{(7)}=\left\langle F_{+}\right\rangle_{(8)}=1$. In the static case, on the other hand, one finds the following expressions:

$\left\langle F_{-}\right\rangle_{(1)}=\sqrt{2}$ 


$$
\begin{aligned}
& \left\langle F_{-}\right\rangle_{(2)}=\sqrt{2} \cos \left(\frac{3 \tau d}{4}\right) \\
& \left\langle F_{-}\right\rangle_{(3)}=1+\cos \left(\frac{3 \tau d}{4}\right) \\
& \left\langle F_{-}\right\rangle_{(4)}=\cos \left(\frac{3 \tau d}{4}\right)+\cos \left(\frac{3 \tau d}{2}\right) \\
& \left\langle F_{+}\right\rangle_{(5)}=-\frac{1}{4}(\sqrt{2}-2)\left[\cos \left(\frac{3 \tau d}{4}\right)+\cos \left(\frac{3 \tau d}{2}\right)\right] \\
& \left\langle F_{+}\right\rangle_{(6)}=\frac{1}{4}\left[1+\cos \left(\frac{3 \tau d}{4}\right)-(\sqrt{2}-1) \cos \left(\frac{3 \tau d}{2}\right)-(\sqrt{2}-1) \cos \left(\frac{9 \tau d}{4}\right)\right] \\
& \left\langle F_{+}\right\rangle_{(7)}=\cos 2\left(\frac{3 \tau d}{8}\right) \cos \left(\frac{3 \tau d}{4}\right) \\
& \left\langle F_{+}\right\rangle_{(8)}=\frac{1}{2} \cos ^{2}\left(\frac{3 \tau d}{8}\right)\left[\cos \left(\frac{3 \tau d}{4}\right)+\cos \left(\frac{3 \tau d}{2}\right)+2 \sin ^{2}\left(\frac{9 \tau d}{8}\right)\right] .
\end{aligned}
$$

The numerical values of these expectation values are given in Table 1 for $d=10 \mathrm{kHz}, \tau_{p}=1 \mu$ s and intervals between pulses $\tau=100 \mu \mathrm{s}-\tau_{p}=99 \mu \mathrm{s}$. Equation 8 reveals that the dipolar interaction is not fully refocused at the end of a DANTE solid echo sequence. The $r f$ pulses in the second refocusing DANTE train have different effects under spinning and static conditions, i.e., in the spinning case, the $r f$ pulses lead to an increase of $\left\langle I_{+}\right\rangle$whereas, in the static case, the $r f$ pulses result in a decrease of SQ coherence. This can be estimated by the variation of the relevant expectation values between points (4) and (5) $\Delta\left\langle F_{+}\right\rangle_{\left(5,4^{\prime}\right)}=0.2929$ and -0.1527 , for spinning and static cases, respectively, or between points (6) and (7) $\Delta\left\langle F_{+}\right\rangle_{(7,6)}=0.7071$ and -0.3491 , for spinning and static cases, respectively. These effects are highlighted by dashed arrows in Fig. 2 (b). Therefore, the descriptor $\Delta\left\langle F_{+}\right\rangle_{(n, n-1)}$ evaluated during $r f$ pulses in the second DANTE train of the solid echo sequence is always positive under MAS and always negative in static samples. During the first DANTE train of the echo sequence on the other hand, $\Delta\left\langle F_{-}\right\rangle_{(n, n-1)}$ is always 
positive during the pulses in both spinning and static samples. Furthermore, and only during the second refocusing train of $r$ pulses, the inhomogeneous dipolar interaction increases the projection onto the transverse plane during free evolution in static samples. This can be appreciated between points (5) and (6), where one finds $\Delta\left\langle F_{+}\right\rangle_{(6,5)}=0.4794$. By contrast, in the intervals between rf pulses, this descriptor always vanishes under MAS, since two consecutive points, say (5) and (6), correspond to tops of rotational echoes, where all inhomogeneities are refocused. In the single-crystal case of Fig. 2(b), and in contrast to what was observed in the powder average of Fig 1(b), the last free evolution interval between (7) and (8) results in $\Delta\left\langle F_{+}\right\rangle_{(8,7)}$ $=-0.0213$. This is in disagreement with what can be seen between points (5) and (6). However, we find that, when extensive powder averaging is performed, the descriptor $\Delta\left\langle F_{+}\right\rangle_{(n, n-1)}$ evaluated in the intervals between $r$ pulses is always positive in static samples during the second train of a DANTE solid echo sequence. This latter feature is ensured by coherence transfer selection and reflects the partial refocusing effect of the second DANTE pulse train.

Figure 3(a) shows an experimental solid-echo spectrum of the protons of water in a static sample of barium chlorate monohydrate $\left(\mathrm{BaClO}_{3} \cdot \mathrm{H}_{2} \mathrm{O}\right)$. An almost ideal Pake pattern is observed, thus confirming, as discussed above, that intermolecular dipolar couplings can be neglected and that the two-spin system can be assumed to be isolated. The asymmetry in the shoulders of the pattern (the low-frequency or right-hand side being more pronounced than the high-frequency side) is due to the shielding anisotropy of the protons. Figures $3(b),(c)$ and (d), show solid echo spectra obtained with different spin-echo sequences $D_{5}^{x}-\tau^{\prime}-D_{5}^{\phi}-\tau^{\prime}-, D_{10}^{x}-\tau^{\prime}-D_{10}^{\phi}-\tau^{\prime}-$ and $D_{20}^{x}-\tau^{\prime}-D_{20}^{\phi}-\tau^{\prime}-$ of the same sample as in Fig $3(\mathrm{a})$. Since the $r f$ field strength was $v_{1}=114$ $\mathrm{kHz}$, the lengths of the individual pulses were chosen to be $\tau_{p}=0.44,0.22$ and $0.11 \mu \mathrm{s}$, corresponding to cumulative nutation angles of $\beta=\pi / 2$ in all cases $(0.44 \times 5=0.22 \times 10=0.11 \times$ $20=2.2 \mu \mathrm{s}$.) The delays between the pulses were $\tau=56.60,31.30$ and $12.10 \mu \mathrm{s}$, respectively, corresponding after Fourier transformation to excitation profiles with $r f$ spikelets spaced by 17.5, 31.9 and $81.9 \mathrm{kHz}$, respectively. The delay between the two DANTE trains was $\tau^{\prime}=0$ in all cases. As previously highlighted by the simulations of Fig. 1, at the end of the solid echo sequences, the DANTE trains indeed allow one to acquire reasonably undistorted lineshapes in static samples. 
These spectra show that, by means of DANTE pulse trains, one can selectively excite 'slices' across an inhomogeneous lineshape. This amounts to say that a chosen 'window' of crystallite orientations within a powder pattern can be selectively excited and explored.

Figure 4 shows experimental spectra analogous to those of Fig. 3, with the exception that a roughly 4-fold weaker $r f$-field strength of $v_{1}=26 \mathrm{kHz}\left(\tau_{90^{\circ}}=9.6 \mu \mathrm{s}\right)$ was used in a solution-state NMR spectrometer. The lower $r f$ amplitude leads to the slightly distorted powder pattern of Fig. 4(a), where the shoulders of the Pake pattern are less pronounced compared to those of Fig. 3(a). On the other hand, the DANTE spectra of Fig. $4(\mathrm{~b}-\mathrm{d})$ obtained with $v_{1}=26 \mathrm{kHz}$ are substantially identical to those of Fig. 3(b-d), obtained $v_{1}=114 \mathrm{kHz}$. Subtle differences in the relative intensities of the peaks of Figs. 3 and 4 are ascribed to variations in $T_{1}$ observed for crystallites of $\mathrm{BaClO}_{3} . \mathrm{H}_{2} \mathrm{O}$ with different orientations [15] bearing in mind that different recovery delays of 5 and $10 \mathrm{~s}$ were used in the experiments of Figs. 3 and 4 . These observations confirm that DANTE echo trains may be used to excite and refocus inhomogeneously broadened lines with line widths that greatly exceed the strength of the $r f$ field.[6]

When dealing with an isolated dipolar-coupled spin pair, as in our case, each single crystal gives rise to a doublet with a splitting determined by the magnitude of the coupling constant $d$ and the angle $\theta$ subtended between the main axis of the dipolar tensor and the external magnetic field. The two transitions of the doublet have frequencies $v_{ \pm}^{D}(\theta)=\delta_{i s o} \pm 3 d\left(3 \cos ^{2} \theta-1\right) / 4$ where $\delta_{\text {iso }}$ is the chemical shift, assumed to be isotropic. It is worth bearing in mind at this point that each single transition of the doublet is 'shared' by each of the two spins. In fact, if one could detect only one of the two protons, one would obtain exactly the same Pake pattern with half the intensity, i.e., $\left\langle F_{ \pm}(t)\right\rangle=2\left\langle I_{ \pm}(t)\right\rangle=2\left\langle S_{ \pm}(t)\right\rangle$. The splitting is maximum when $\theta=0^{\circ}$, i.e., $\Delta v^{D}(0)=3 d$, vanishes at the magic angle $\theta=54.7^{\circ}$, and becomes negative for $\theta=90^{\circ}$, $\Delta v^{D}(\pi / 2)=-3 d / 2$. Between the inner 'horns' of the pattern, each doublet can correspond to two different orientations. By contrast, outside these 'horns', near the 'shoulders', each doublet corresponds to a crystallite with a unique orientation. One can choose the parameters of a DANTE static echo sequence so as to obtain spectra that correspond either to a unique or to two crystal 
orientations by setting the carrier frequency $v_{r f}$ and the delay $\tau$ between adjacent pules according to:

$$
\begin{aligned}
& v_{r f}(\theta)=v_{ \pm}^{D}(\theta)=\delta_{i s o} \pm \frac{3}{4} d\left(3 \cos ^{2} \theta-1\right) \\
& \tau(\theta)^{-1}=\frac{3}{2} d\left(3 \cos ^{2} \theta-1\right)
\end{aligned}
$$

A system made up of a single isolated spin $S=1 / 2$, subject to an anisotropic interaction such as a chemical shift anisotropy, would produce a powder lineshape made up of a single peak (rather than a doublet) for each crystal orientation. In such cases, each peak resulting from a DANTE echo can be ascribed to a single crystallite orientation. Thus DANTE-based techniques allow one to detect a subspectrum corresponding to a particular range of crystallite orientations. In spectra such as those shown in Fig. 3(b-d) and 4(b-d), one expects a range of crystallite orientations to contribute to the signal. The 'purity' of DANTE signals in terms of the number of crystallites that contribute depends on the width of the $r f$ spikelets resulting from the DANTE trains. This width is determined by the total length of the $r$ sequence and, in the case of the solid echoes used in this work, is proportional to $(2 M \tau)^{-1}$.

Figure 5 shows spectra which demonstrate how DANTE echoes may be tuned so as to explore particular sets of crystallite orientations. In Fig. 5(a), the static pattern obtained with a conventional solid echo is shown to guide the eye. Figure 5(b) shows that a 'horns-only' DANTE spectrum may be obtained by an appropriate choice of the carrier frequency and pulse interval. Figure 5(c) shows that a 'single-horn' spectrum may be obtained simply by decreasing the pulse intervals used in (b). Figure 5(d) shows a 'shoulders-only' spectrum of the Pake pattern of Fig. 5(a). Near the shoulders, doublets associated with single-crystallite orientations may be explored. A 'single-shoulder' spectrum is shown in Fig. 5(e). In the latter case, a single transition of a doublet associated with a single crystal is excited. The carrier frequency is indicated by black arrows when different from $0 \mathrm{~Hz}$. 
The necessity to resort to a second DANTE train for refocusing is demonstrated in Figure 6 . In (a), a spectrum obtained with a $D_{10}^{x}-\tau^{\prime}-D_{10}^{\phi}-\tau^{\prime}-$ echo is shown. This is in very good agreement with the simulation of Fig. 1(i). Figure $6(\mathrm{~b})$ shows the spectrum resulting from a single $D_{10}^{x}-\tau^{\prime}$-train, i.e., when no refocusing train is used. The lineshape obtained in the latter case is characterized by distortions that are in very good agreement with the simulations shown in Fig. $1(f)$. The partial failure to refocus the dipolar interactions in static samples at the end of a DANTE solid echo sequence results in distortions of the spectra that are analogous to those obtained if one inserts a long 'dead time' before starting signal acquisition. However, as may be appreciated in the experimental spectra of Figures $3(b-d)$ and $4(b-d)$, the extent of dephasing is perfectly acceptable in our case.

\section{Conclusions}

We have analyzed the response to DANTE trains of $r f$ pulses applied to static samples in solid-state NMR spectroscopy. In contrast to MAS experiments, where it is possible to use a single rotor-synchronized DANTE train for direct excitation without resorting to refocusing, in static samples one can only obtain reasonably undistorted lineshapes if one uses spin echoes that are refocused by a second DANTE pulse train. This has been shown both by numerical simulations and by experiments performed on the protons of hydration water in barium chlorate monohydrate. The spectral bandwidths that one can access with DANTE techniques in static samples open up new ways for exploring the responses of selected crystallites in powder samples.

\section{Experimental details}

A sample of ca. $50 \mathrm{mg} \mathrm{Ba}\left(\mathrm{ClO}_{3}\right)_{2} \cdot \mathrm{H}_{2} \mathrm{O}$ (Alfa Aesar) was kept under vacuum (ca. $10 \mathrm{~Pa}$ ) overnight prior to the experiments in order to minimize the adsorbtion of water which leads to a sharp isotropic peak in the middle of the Pake pattern. All ${ }^{1} \mathrm{H}$ spectra of Figure 3 excited with "hard" rf pulses have been recorded at the EPFL on a Bruker 400 spectrometer (9.4 T) with an Avance-II console, using $3.2 \mathrm{~mm}$ rotors in a triple resonance MAS probe designed for lowtemperature measurements near $100 \mathrm{~K}$, but used at room temperature without microwave irradiation and without spinning. The $r f$-field amplitude was $v_{1}=114 \mathrm{kHz}$. A recycling delay of $5 \mathrm{~s}$ 
was used. All ${ }^{1} \mathrm{H}$ spectra of Figures 4, 5 and 6 excited with "soft" $r f$ pulses have been recorded at the University of Neuchâtel on a Bruker 400 spectrometer (9.4 T) with an Avance-II console, using $5 \mathrm{~mm}$ tubes in a triple resonance probe designed for solution-state NMR. The $r f$-field amplitude was $v_{1}=26 \mathrm{kHz}$. A recycling delay of $10 \mathrm{~s}$ was used. All solid echoes were phase cycled for the selection of the $p=0 \rightarrow p=1 \rightarrow p=-1$ pathway by nesting Exorcycle [16] and Cyclops [17] to produce an overall 16-step phase cycle. The delay between the two DANTE trains was $\tau^{\prime}=0$, in all cases. Numerical simulations were performed with SIMPSON,[18] using 2000 crystal orientations sampled with the REPULSION scheme.[19]

\section{Acknowledgements}

This work was supported by the Swiss National Science Foundation (SNSF), the Ecole Polytechnique Fédérale de Lausanne (EPFL), the University of Neuchâtel, the Neuchâtel Platform of Analytical Chemistry (NPAC), the Swiss Commission for Technology and Innovation (CTI) and the European Research Council (ERC, project 'Dilute para-water').

\section{References}

[1] M.J. Duer, Solid-State NMR Spectroscopy: Principles and Applications, Blackwell Science, UK, 2002.

[2] G. Bodenhausen, R. Freeman and G.A. Morris, J. Magn. Reson. 23 (1976) 171.

[3] P. Caravatti, G. Bodenhausen and R.R. Ernst, J. Magn. Reson. 55 (1983) 88.

[4] V. Vitzthum, M.A. Caporini, S. Ulzega and G. Bodenhausen, J. Magn. Reson. 212 (2011) 234.

[5] V. Vitzthum, M.A. Caporini, S. Ulzega, J. Trébosc, O. Lafon, J.-P. Amoureux and G. Bodenhausen, J. Magn. Reson. 223 (2012) 228.

[6] D. Carnevale, V. Vitzthum, O. Lafon, J. Trébosc, J.-P. Amoureux and G. Bodenhausen, Chem. Phys. Lett. 553 (2012) 68.

[7] D. Carnevale, A.J. Perez Linde, G. Bauer and G. Bodenhausen, Chem. Phys. Lett. 580 (2013) 172.

[8] X. Lu, J. Trébosc, O. Lafon, D. Carnevale, S. Ulzega, G. Bodenhausen and J.-P. Amoureux, J. Magn. Reson. 236 (2013) 105.

[9] D. Carnevale, S.E. Ashbrook and G. Bodenhausen. RSC Adv. 4 (2014) 56248. 
[10] G.E. Pake, J. Chem. Phys. 16, 4 (1948) 327.

[11] J.G. Powles and P. Mansfield, Phys. Lett. 2 (1962) 58.

[12] J.G. Powles and J.H. Strange, Proc. Phys. Soc. 82 (1963) 6.

[13] S. Antonijevic and S. Wimperis, J. Magn. Reson. 164 (2003), 343.

[14] G. Bodenhausen, H. Kogler and R.R. Ernst, J. Magn. Reson. 58 (1984) 370.

[15] J.R. Long, R. Ebelhäuser and R.G. Griffin, J. Phys. Chem. A 101 (1997) 988.

[16] G. Bodenhausen, R. Freeman and D.L. Turner, J. Magn. Reson. 27 (1977) 511.

[17] D.I. Hoult and R.E. Richards, Proc. Roy. Soc. (London) A344 (1975) 311.

[18] M. Bak, J.T. Rasmussen and N.C. Nielsen, J. Magn. Reson. 147 (2000) 296.

[19] M. Bak and N.C. Nielsen, J. Magn. Reson. 125 (1997) 132.

Table 1 Expectation values of relevant operators at selected points in the pulse sequence $D_{2}^{x}-\tau^{\prime}-D_{2}^{\phi}-\tau^{\prime}-$ of Fig. 2, evaluated using Equations (2-8). The relevant parameters are $d=-10 \mathrm{kHz}, v_{1}=125 \mathrm{kHz}, \tau_{p}=1 \mu \mathrm{s}, \tau=100 \mu \mathrm{s}-\tau_{p}=99 \mu \mathrm{s}$ and $\tau^{\prime}=0$. Pulses are considered as ideal with a vanishing duration. A single crystal is assumed, with the principal axis of the dipolar tensor perpendicular to the external magnetic field $B_{0}$.

\begin{tabular}{ccc}
\hline$\left\langle F_{ \pm}\right\rangle_{(n)}$ & Spinning & Static \\
\hline$(1)$ & $\sqrt{2}$ & $\sqrt{2}$ \\
$(2)$ & $\sqrt{2}$ & -0.0667 \\
$(3)$ & 2 & 0.9529 \\
$(4)$ & 2 & -1.0427 \\
$\left(4^{\prime}\right)$ & 0 & 0 \\
$(5)$ & $1-1 / \sqrt{2}$ & -0.1527 \\
$(6)$ & $1-1 / \sqrt{2}$ & 0.3267 \\
$(7)$ & 1 & -0.0224 \\
$(8)$ & 1 & -0.0437 \\
\hline
\end{tabular}


(a)

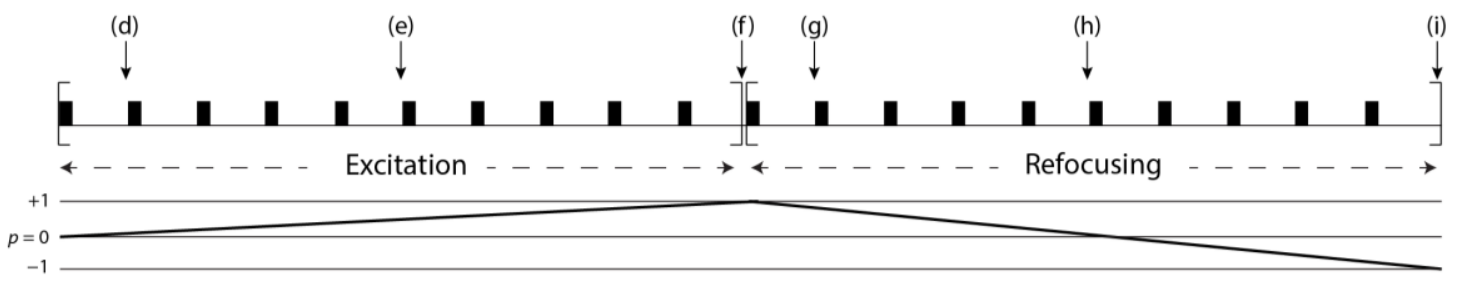

(b)

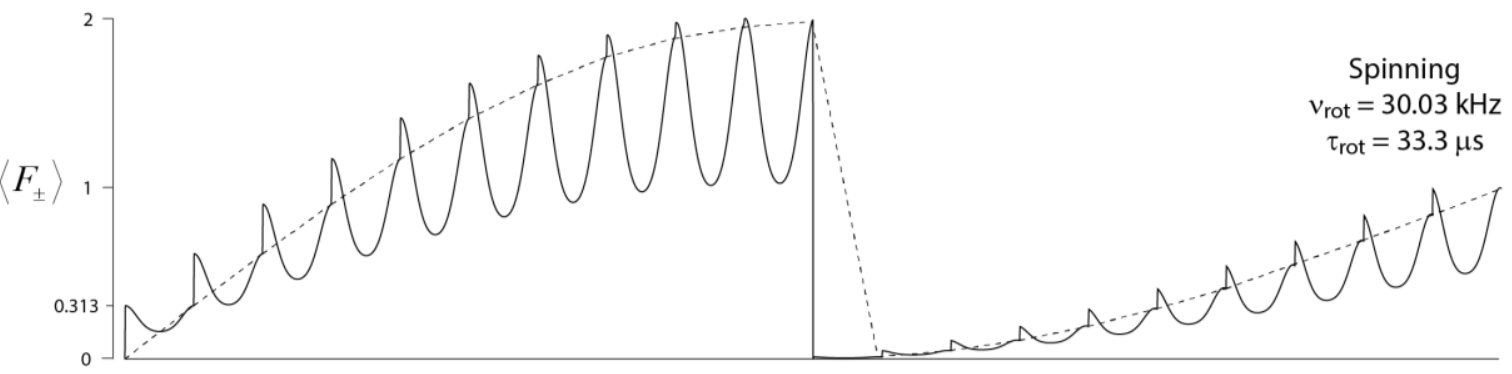

(c)

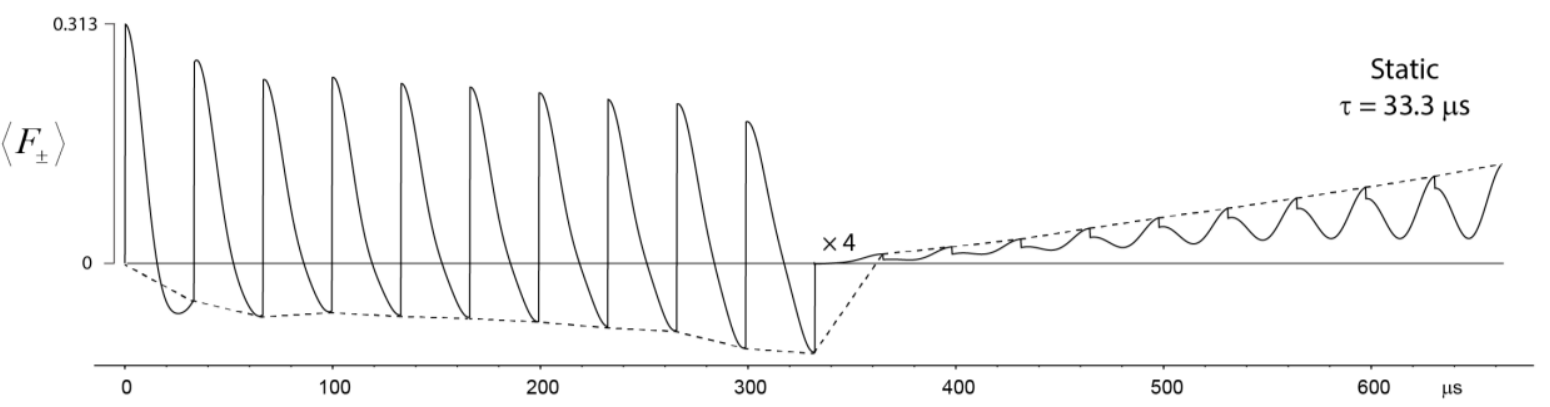

(d)

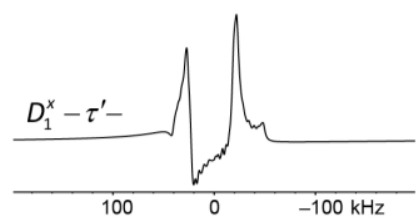

(g)

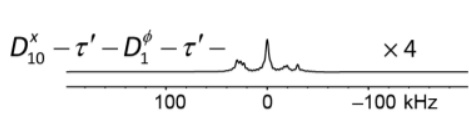

(e)

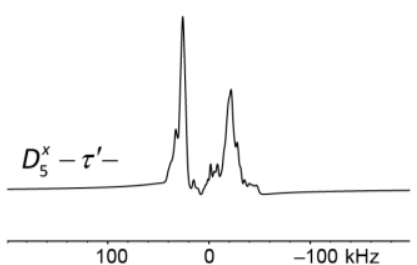

(h)

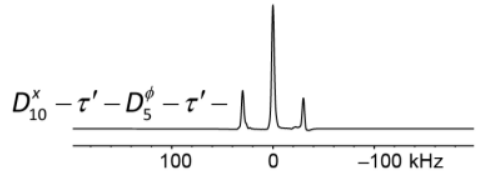

(f)

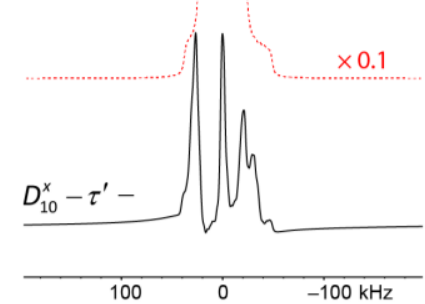

(i)

Figure 1 (a) Scheme representing a solid echo experiment comprising two $D_{10}$ DANTE sequences for excitation and refocusing, i.e., either a $D_{1}^{10, x}-\tau^{\prime}-D_{1}^{10, \phi}-\tau^{\prime}-$ or $D_{10}^{x}-\tau^{\prime}-D_{10}^{\phi}-\tau^{\prime}-$ 
(with $\tau^{\prime}=0$ ) for spinning or static samples, respectively, with corresponding coherence transfer pathway (CTP) diagram. (b) and (c) Numerical simulations of the nutation of the magnetization during a $D_{1}^{10, x}-\tau^{\prime}-D_{1}^{10, \phi}-\tau^{\prime}-$ and $D_{10}^{x}-\tau^{\prime}-D_{10}^{\phi}-\tau^{\prime}-$ solid echo sequences, for static and spinning samples, respectively. Dashed black lines indicate signal intensities when the acquisition is started at the end of each DANTE block in the course of the pulse sequence of (a). (d)-(i) Simulated spectra of spinning samples obtained by Fourier transformation of signals when the acquisition is started at the points indicated by black arrows in (a). The red lines in ( $f$ ) and (i) show the ideal powder pattern of the static solid. The relevant parameters of the system with two equivalent dipolar-coupled protons are $d=-30$ $\mathrm{kHz}, v_{1}=125 \mathrm{kHz}, \tau_{p}=0.2 \mu \mathrm{s}, \tau^{\prime}=0$ and $\tau=33.1 \mu \mathrm{s}$. A chemical shift anisotropy characterized by $\delta_{\text {iso }}=0 \mathrm{ppm}, \Delta_{\text {aniso }}=-10 \mathrm{ppm}$ and $\eta=0.9$ was considered. The external field was $B_{0}=9.4 \mathrm{~T}$ and the principal components of the shielding and dipolar tensors were assumed to be parallel to one another. Powder averaging was performed over 2000 crystal orientations. Only the real parts of the echoes and spectra are shown. The spectra (d-f) result from the acquisition of $p=-1$ coherence. The first half of the nutation profiles of (b) and (c) result from the acquisition of $p=+1$ coherence, in accordance with the coherence transfer pathway of (a). The second half of the nutation profile of (c) has been expanded vertically by a factor 4 for clarity. 
(a)

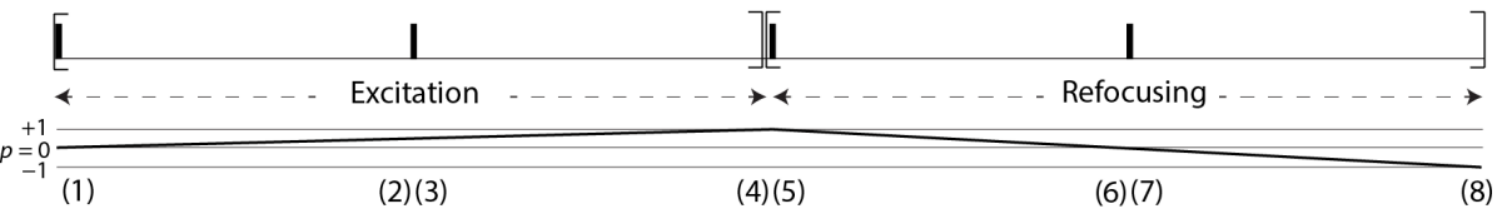

(b)

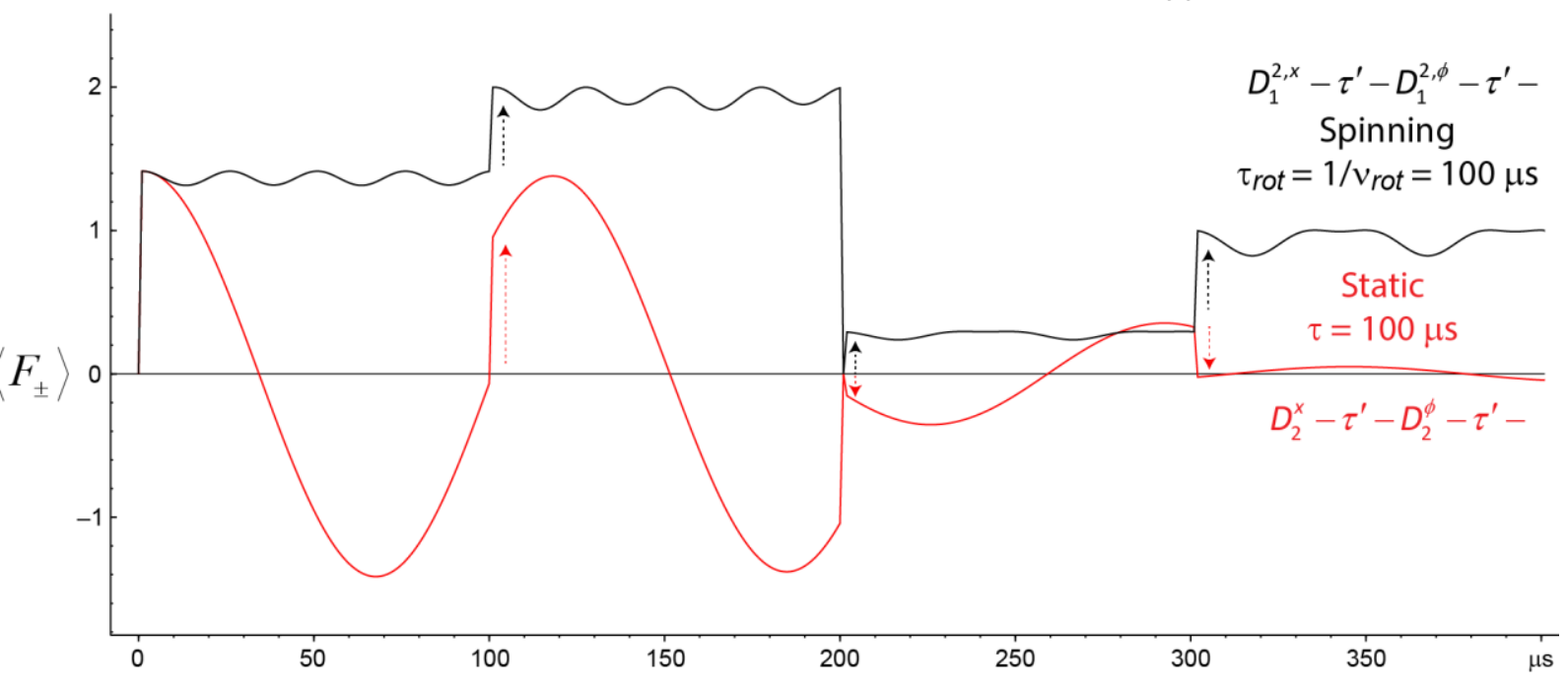

Figure 2 (a) Scheme representing a solid echo experiment comprising two $D_{2}$ DANTE sequences, with corresponding coherence transfer pathway diagram. (b) Numerical simulations of the experiment shown in (a) for spinning $\left(D_{1}^{2, x}-\tau^{\prime}-D_{1}^{2, \phi}-\tau^{\prime}-\right)$ or static samples ( $\left.D_{2}^{x}-\tau^{\prime}-D_{2}^{\phi}-\tau^{\prime}-\right)$, in black and red, respectively. The relevant parameters of the system consisting of two equivalent dipolar-coupled ${ }^{1} \mathrm{H}$ nuclei are $d=-10 \mathrm{kHz}, v_{1}=125 \mathrm{kHz}, \tau_{p}=1 \mu \mathrm{s}$, $\tau=100 \mu \mathrm{s}$ and $\tau^{\prime}=0$. All pulses are considered to be ideal. Simulations were performed assuming one single crystal, with a dipolar tensor perpendicular to the external magnetic field $B_{0}$. Only the real parts of the relevant expectation values are shown. 
(a)

(b)
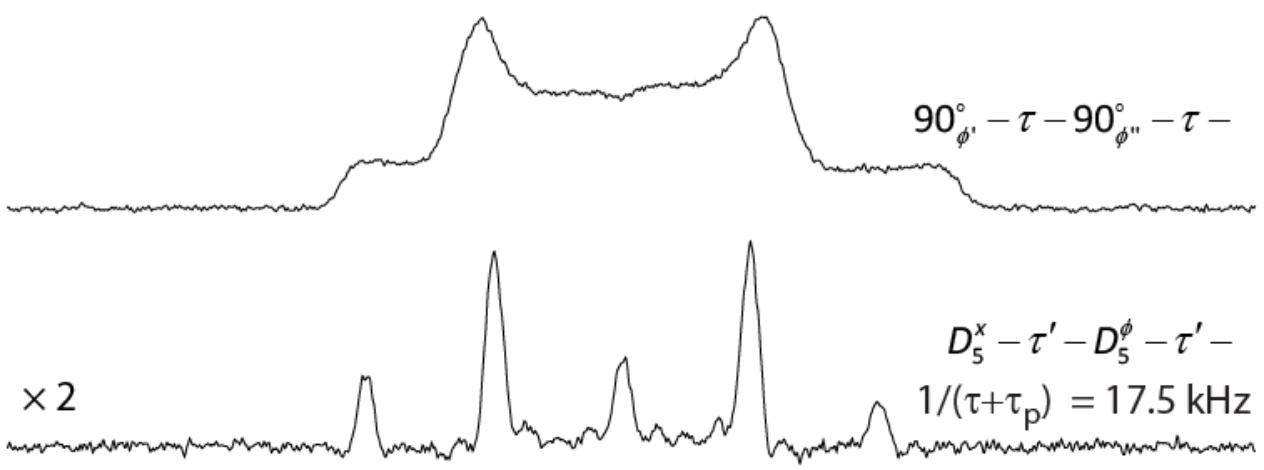

(c)

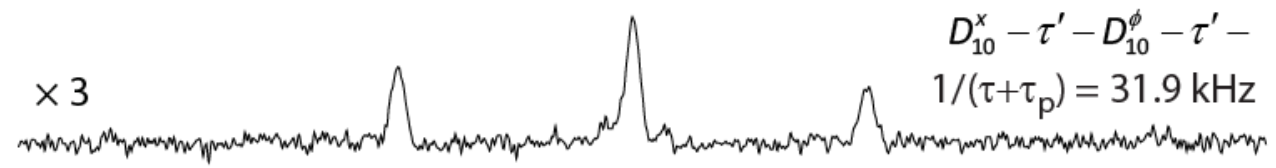

(d)

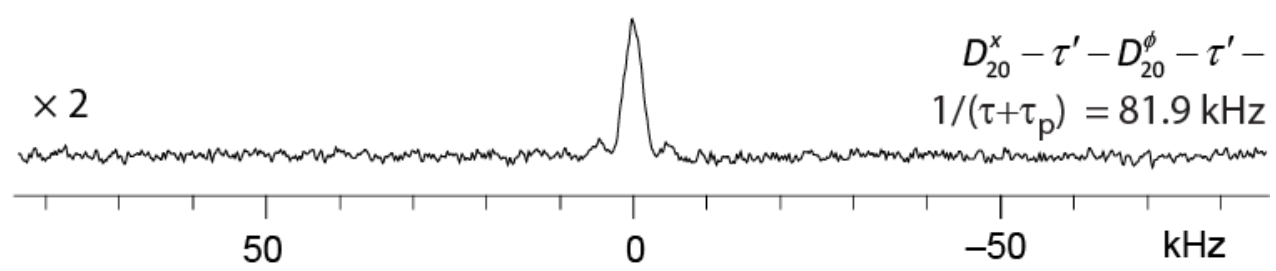

Figure 3 (a) Experimental static lineshape due to pairs of dipolar-coupled protons in a static nonspinning sample of barium chlorate monohydrate, obtained by Fourier transformation of the decaying half of a solid echo excited by a conventional $90^{\circ}-\tau-90^{\circ}-\tau$ sequence with $90^{\circ}$ pulses of $2.2 \mu$ s duration and $\tau=50 \mu$ s. (b-d) Spectra obtained by Fourier transformation of the decaying halves of solid echoes excited by DANTE sequences (b) $D_{5}^{x}-\tau^{\prime}-D_{5}^{\phi}-\tau^{\prime}-$, (c) $D_{10}^{x}-\tau^{\prime}-D_{10}^{\phi}-\tau^{\prime}-$ and (d) $D_{20}^{x}-\tau^{\prime}-D_{20}^{\phi}-\tau^{\prime}-$ with $\tau^{\prime}=0, \tau+\tau_{p}=57.04,31.52$ and $12.21 \mu \mathrm{s}$, respectively. The pulse lengths were $\tau_{p}=0.44,0.22$ and $0.11 \mu \mathrm{s}$, respectively, with a strong $r$-field $v_{1}=114 \mathrm{kHz}$. 
(a)

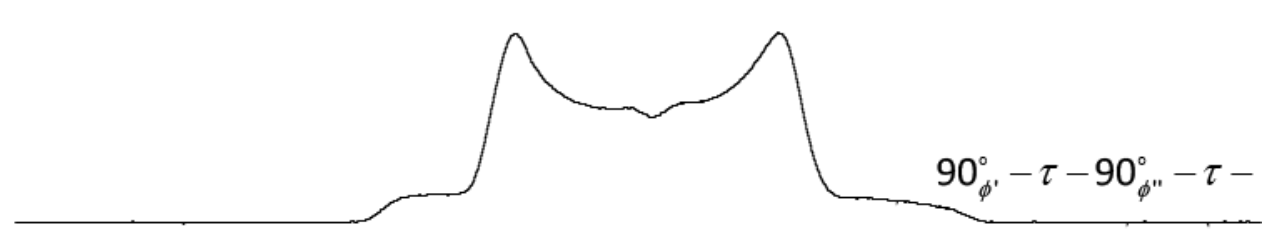

(b)

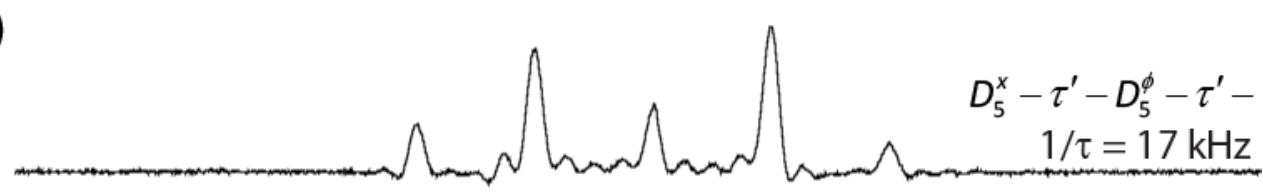

(c)

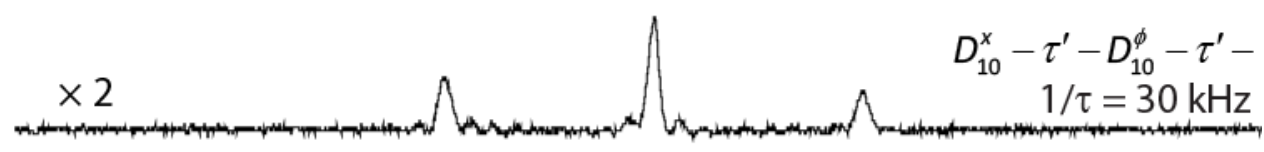

(d)

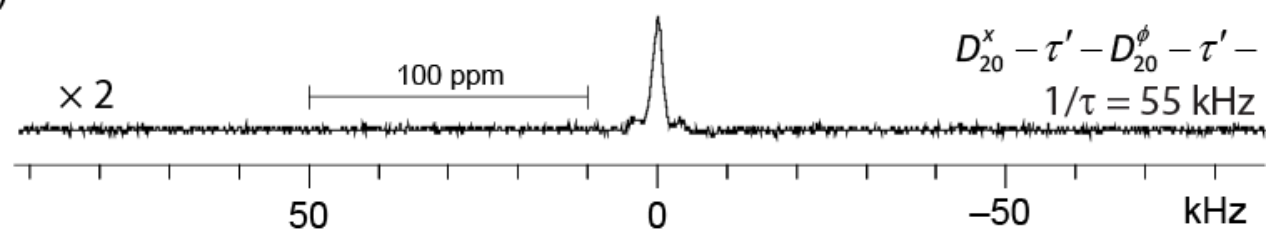

Figure 4 (a) Experimental static lineshape due to pairs of dipolar-coupled protons in a static nonspinning sample of barium chlorate monohydrate, obtained by Fourier transformation of the decaying half of a solid echo excited by a conventional $90_{x}^{\circ}-\tau-90_{\phi}^{\circ}-\tau-$ sequence with $90^{\circ}$ pulses of $9.6 \mu$ s duration and $\tau=50 \mu \mathrm{s}$. (b-d) Spectra obtained by Fourier transformation of the decaying halves of solid echoes excited by DANTE sequences (b) $D_{5}^{x}-\tau^{\prime}-D_{5}^{\phi}-\tau^{\prime}-$ , (c) $D_{10}^{x}-\tau^{\prime}-D_{10}^{\phi}-\tau^{\prime}-$ and (d) $D_{20}^{x}-\tau^{\prime}-D_{20}^{\phi}-\tau^{\prime}-$ with $\tau^{\prime}=0$, and $\tau+\tau_{p}=58.82,33.33$ and $18.18 \mu \mathrm{s}$, respectively. The pulse lengths were $\tau_{p}=1.92,0.96$ and $0.48 \mu \mathrm{s}$, respectively, with a weak rf-field strength $v_{1}=26 \mathrm{kHz}$. The recycling delay was $10 \mathrm{~s}$ in all cases. 16 transients were averaged in (a) whereas 32 scans were acquired in (b-d). The carrier frequency was set to $0 \mathrm{~Hz}$ in all cases. No line broadening was applied. 
(a)

(b)

(c)
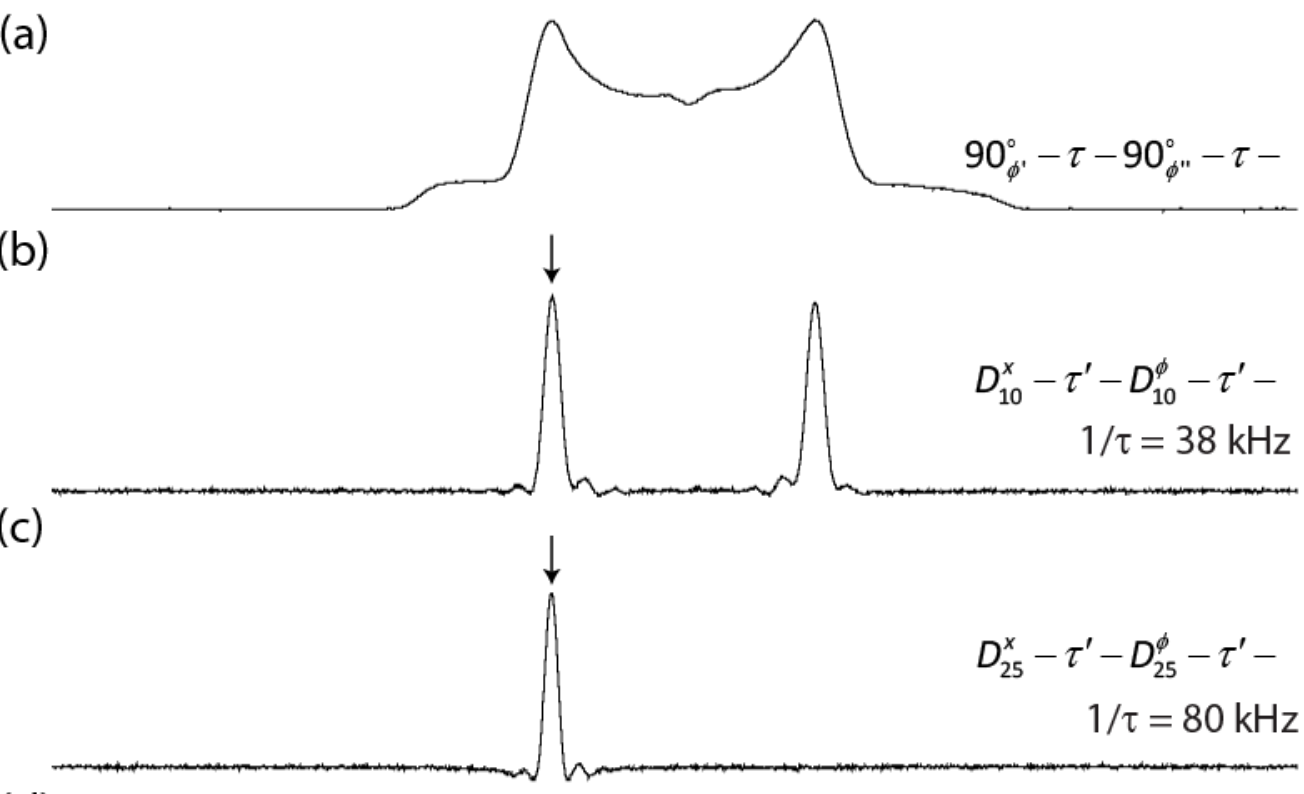

(d)

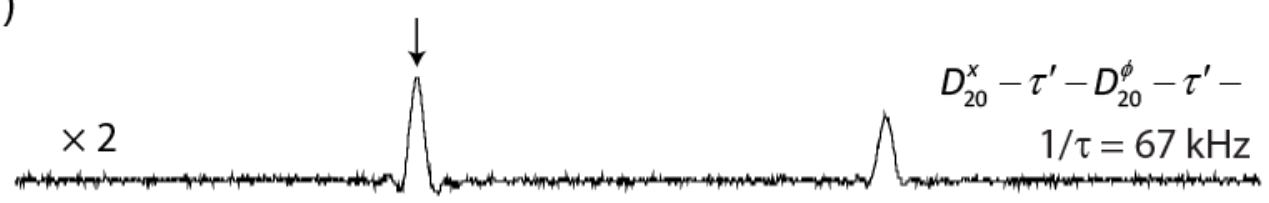

(e)

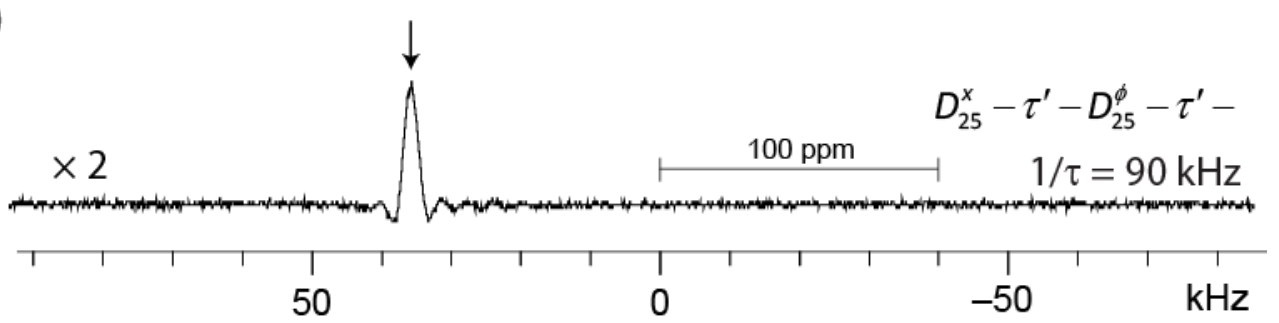

Figure 5 (a) Experimental static lineshape as in Fig. 3(a) shown for comparison. (b-e) Spectra obtained by Fourier transformation of the decaying halves of solid echoes excited by DANTE sequences (b) $D_{5}^{x}-\tau^{\prime}-D_{5}^{\phi}-\tau^{\prime}-$, (c) $D_{10}^{x}-\tau^{\prime}-D_{10}^{\phi}-\tau^{\prime}-$, (d) $D_{20}^{x}-\tau^{\prime}-D_{20}^{\phi}-\tau^{\prime}-$ and (e) $D_{25}^{x}-\tau^{\prime}-D_{25}^{\phi}-\tau^{\prime}-$ with $\tau^{\prime}=0$, and $\tau+\tau_{p}=26.32,12.50,14.93$ and $11.11 \mu$ s, respectively. The pulse lengths were $\tau_{p}=0.96,0.38,0.48$ and $0.38 \mu$ s, respectively, with a weak $r$-field $v_{1}$ $=26 \mathrm{kHz}$. The recycling delay was $10 \mathrm{~s}$ in all cases. 16 transients were averaged in (a) whereas 32 scans were acquired in (b-e). The carrier frequency was set to $0 \mathrm{~Hz}$ in (a) and is indicated by black arrows in all other cases. No line broadening was applied. 

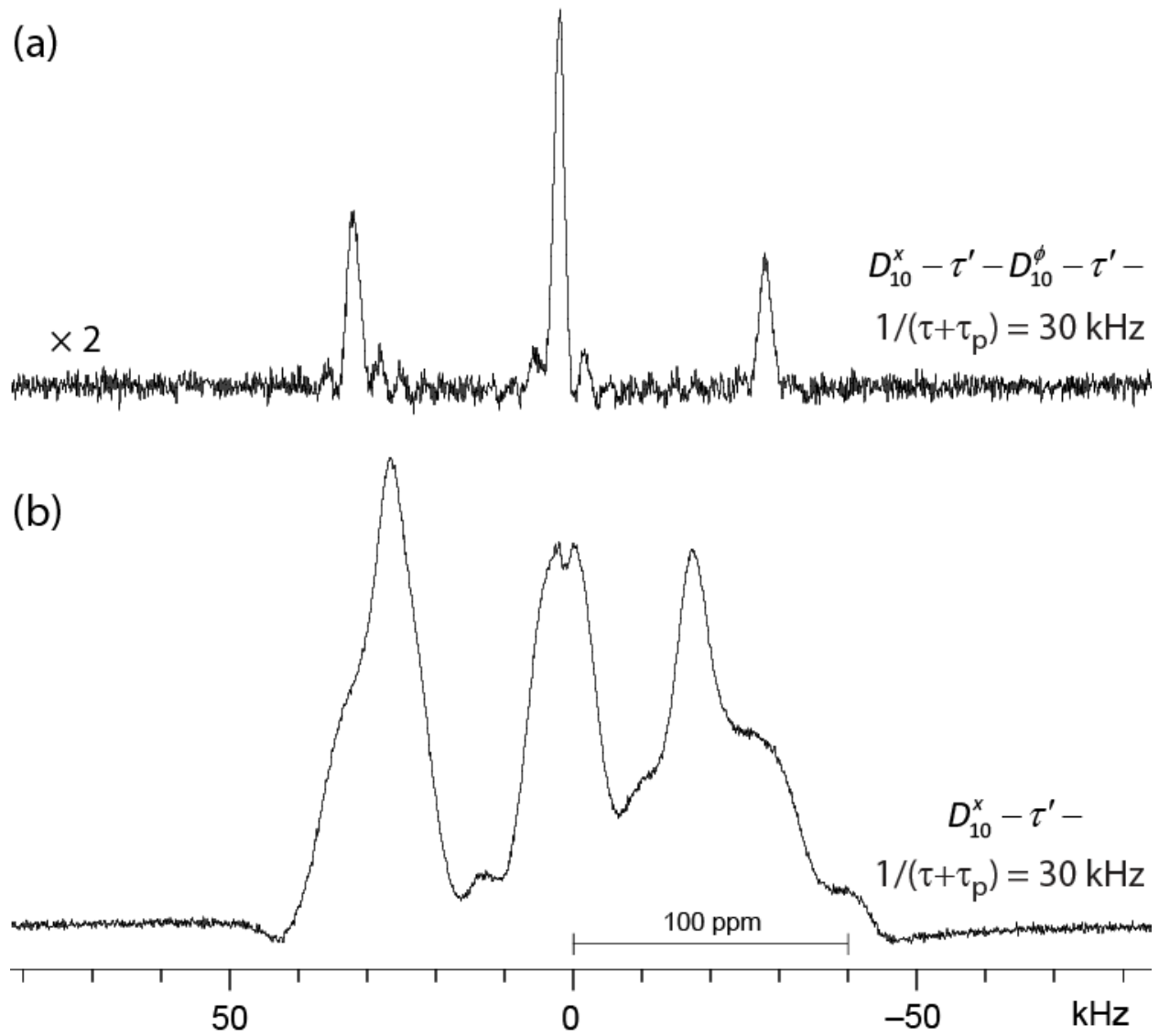

Figure 6 (a) Experimental static lineshape obtained with a sequence $D_{10}^{x}-\tau^{\prime}-D_{10}^{\phi}-\tau^{\prime}-$ as in Fig. 4(c) shown for comparison. (b) Experimental spectrum resulting from a single DANTE sequence $D_{10}^{x}-\tau^{\prime}-$ used for direct excitation, without resorting to a second $D_{10}^{\phi}-\tau^{\prime}-$ train for refocusing, with $\tau+\tau_{p}=33.33 \mu \mathrm{s}, \tau^{\prime}=0$ and $\tau_{p}=0.96 \mu \mathrm{s}$, with a weak $r f$-field $\nu_{1}=26 \mathrm{kHz}$. The recycling delay was $10 \mathrm{~s}$ and 16 transients were averaged in all cases. The carrier frequency was set to $0 \mathrm{~Hz}$ in both (a) and (b). No line broadening was applied. Zero-th and first-order phase corrections were -303 and 308 , and -288 and $308^{\circ}$, in (a) and (b), respectively. 